\title{
Spatially Resolved Micro-Luminescence from GaN/AlGaN Quantum Dots
}

\author{
B. Chwalisz*, A. WysmoŁek, R. Bożek, R. Stępniewski, \\ K. PakuŁa, P. Kossacki, A. Golnik and J.M. Baranowski \\ Institute of Experimental Physics, Warsaw University, Warsaw, Poland
}

We report on the optical experiments performed on low density GaN/AlGaN quantum dots grown on sapphire substrate using SiN during the growth process. The existence of quantum dots in the investigated structures was confirmed by atomic force microscopy. Although macro-luminescence of the investigated structures consist of broad emission lines the micro-photoluminescence experiments performed with the spatial resolution of $0.25 \mu \mathrm{m}$ revealed sharp emission lines from the individual quantum dot in the energy range of $3.20-3.55 \mathrm{eV}$. It is shown that the magnetic fields up to $7 \mathrm{~T}$ do not influence significantly the electronic states of the dots.

PACS numbers: 78.55.Cr, 78.66.Fd, 78.67.Hc, 75.75.+a, 68.65.Hb

\section{Introduction}

The investigation of quantum dots (QDs) grown on wide band-gap materials has attracted much attention driven by applications in optoelectronic devices working in UV. Moreover, the reduced dimensionality of the novel semiconductor structures leads to the observation of new and intriguing phenomena. The dot structures based on GaN have been successfully fabricated using $\mathrm{Si}$ as a surfactant $[1,2]$ or directly on $6 \mathrm{H}-\mathrm{SiC}$ substrates $[3,4]$. Here we report on the new method of fabrication of low density GaN/AlGaN QDs using SiN during the growth process by metalorganic chemical vapour deposition (MOCVD). Micro-photoluminescence ( $\mu$-PL) experiments allowed us to select emission from single QDs. Spatial distribution of the $\mu$-PL correlates with the result obtained using atomic force microscopy (AFM).

* corresponding author; e-mail: barbara.chwalisz@fuw.edu.pl 


\section{Experimental details}

The investigated structure was grown by MOCVD technique using sapphire as a substrate. After growing of AlGaN buffer layer the growth process was interrupted and intentionally SiN layer was deposited using silane and ammonia as gas sources. It was found that the GaN epilayers grown on the GaN/SiN buffer showed some improvement in structural and optical properties $[5,6]$. In the case of the process described here, as a result of the SiN treatment, GaN layer deposited afterwards does not settle as a two-dimensional layer, but grows in a three-dimensional mode, forming small islands. Then GaN structure was covered by AlGaN barrier. This method gives the possibility to create GaN islands of different size with tailored electronic properties.

Micro-photoluminescence experiments were performed using a specially designed [7] microscope objective. The sample was immersed in super-fluid helium $(T=1.6 \mathrm{~K})$ in the cryostat of a superconducting magnet working up to $7 \mathrm{~T}$. PL mapping of a $5 \times 5 \mu \mathrm{m}^{2}$ area was accomplished by rotating a plate placed between the sample and the first lens of the optical system around two perpendicular axes [8]. The exciting laser beam was introduced in a way assuring a focalisation to a spot smaller than $0.3 \mu \mathrm{m}$ in diameter. In both macro- and micro-luminescence experiments $325 \mathrm{~nm}$ line of $\mathrm{He}-\mathrm{Cd}$ laser was employed as an excitation source.

\section{Results and discussion}

Representative, low temperature macro- and $\mu$-PL spectra taken at zero magnetic fields are shown in Fig. 1. The macro-PL spectrum is composed of broad band in the vicinity of the GaN band gap energy. In contrary, $\mu$-PL shows well-resolved sharp emission lines in the energy range of $3.18-3.52 \mathrm{eV}$. We associate them as coming from individual GaN islands embedded by AlGaN barriers. Usually the broad PL emission of ensembles of QDs was observed [1, 4]. Here, typical full width at half maximum (FWHM) of individual line is $\sim 2 \mathrm{meV} . \mu$-PL spectra of

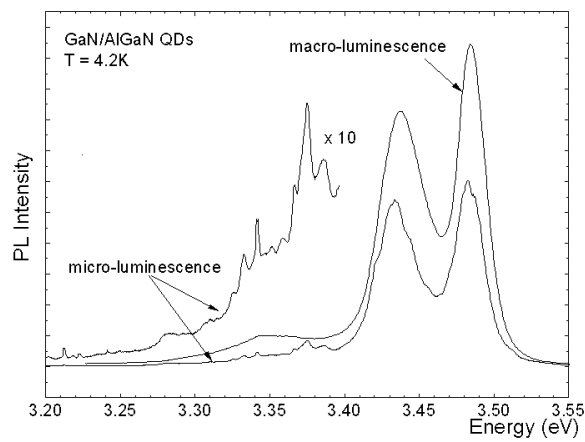

Fig. 1. Macro- and micro-photoluminescence spectra of GaN/AlGaN QDs. 


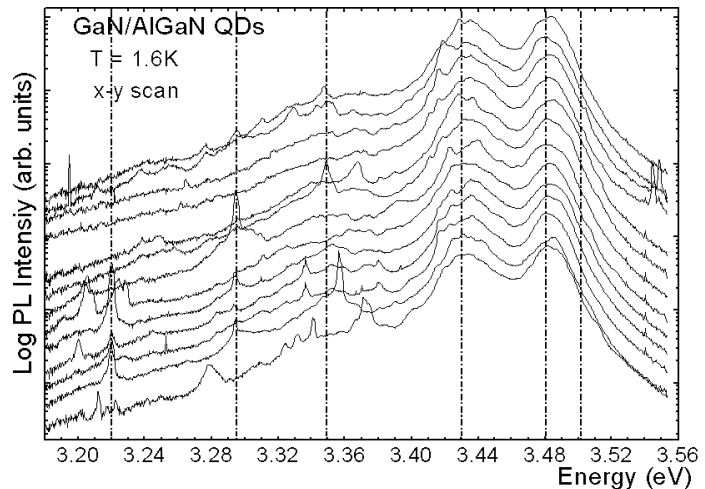

Fig. 2. Micro-PL spectra of GaN/AlGaN QDs collected at various places on the sample.
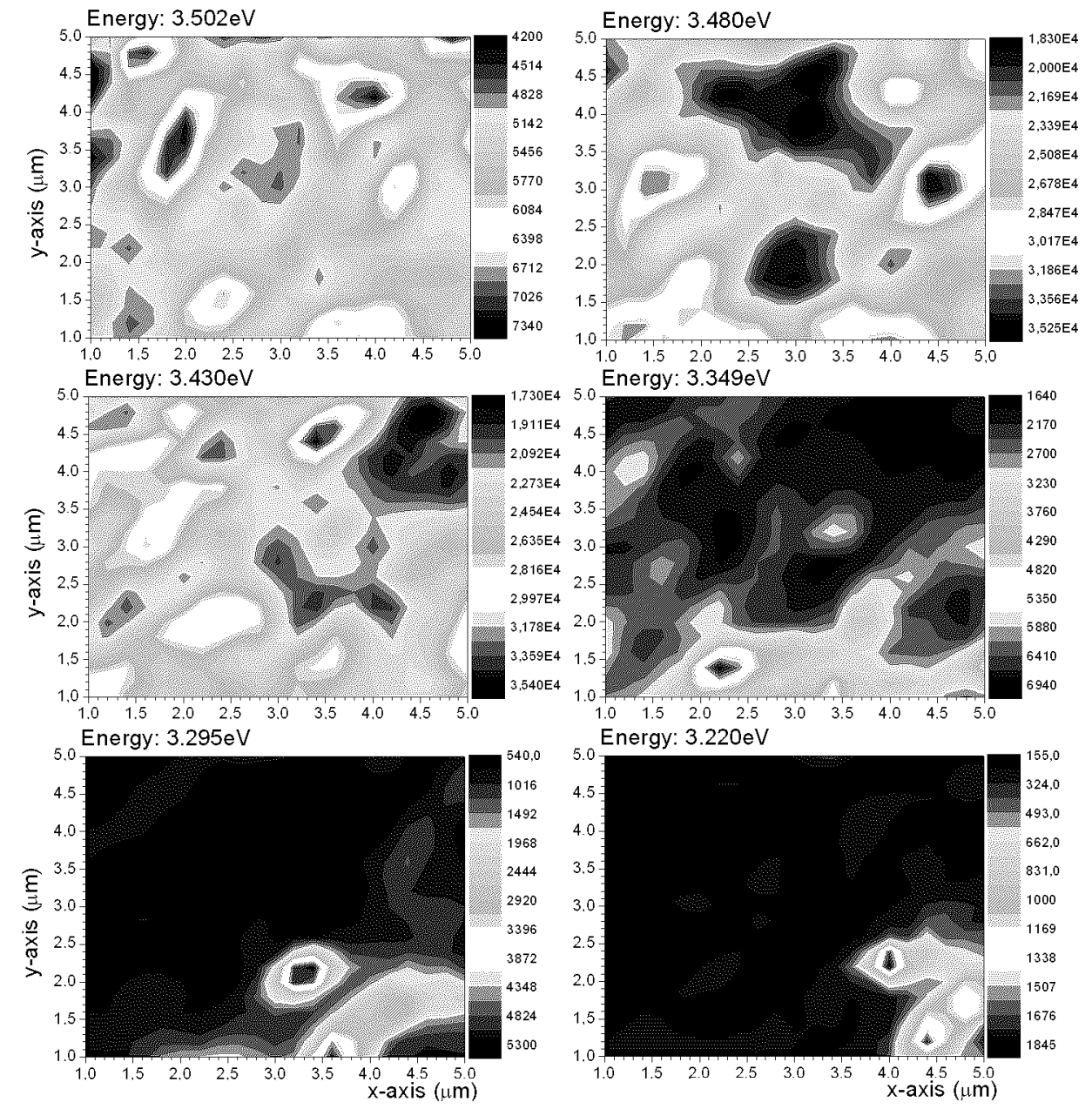

Fig. 3. Micro-PL intensity maps for the emission energies as marked in the figure. 
different sample spots are shown in Fig. 2. Figure 3a-f illustrates the $5 \times 5 \mu \mathrm{m}^{2}$ PL intensity maps for the emission energies as marked in Fig. 2. From the intensity maps it can be directly seen that luminescence detected at specified energy is limited to the strictly defined sample region, different for diverse energies. On the basis of intensity changes (Fig. 3) the diameter of the smallest objects can be evaluated to be smaller than experimental resolution, $0.25 \mu \mathrm{m}$.

The wide spectral range of the emission energy is probably caused by variation in the size of the dots and changes in the localizing potential. Moreover, in GaN/AlGaN structures the energy variation is enhanced by the existence of strong build-in electric fields, which lead to spatial separation and substantial energy shifts of the corresponding levels of electrons and holes in the QDs.

The results obtained from $\mu$-PL can by compared with those obtained by AFM. Figure $4 \mathrm{a}$ and b illustrates the typical AFM images of $5 \times 5 \mu \mathrm{m}^{2}$ sample surface. The extracted average island height is approx. $200 \mathrm{~nm}$ and the diameter up to approx. $500 \mathrm{~nm}$. It is supposed that the actual dimensions of the dots are smaller, since the $150 \mathrm{~nm} \mathrm{AlGaN} \mathrm{cap} \mathrm{layer} \mathrm{covering} \mathrm{dots} \mathrm{influences} \mathrm{the} \mathrm{picture}$ observed in AFM effectively enlarging the underlying dot structures. The obtained dimensions are similar to that obtained from $\mu$-PL experiments. The dot density in the sample is approx. $2 \times 10^{8} \mathrm{~cm}^{-2}$, which is a rather small value for GaN QDs $[1,2,6,9]$.

(a)

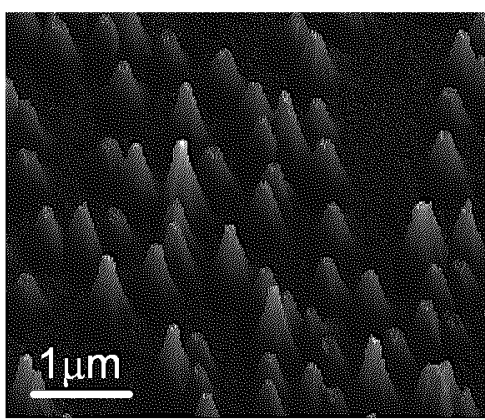

(b)

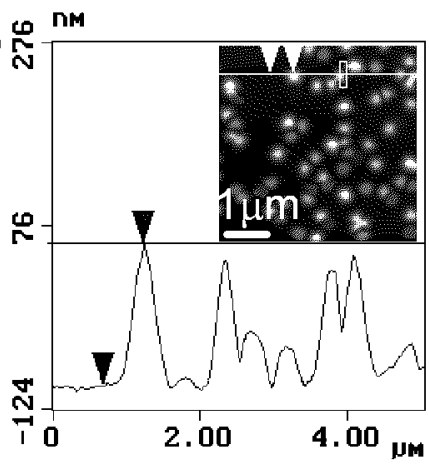

Fig. 4. AFM images of the $5 \mu \mathrm{m}^{2} \mathrm{GaN} / \mathrm{AlGaN}$ QDs surface morphology, (a) bird's eye view, (b) plan view and cross-section.

To investigate the quantum efficiency from the QDs the excitation power dependence on $\mu$-PL was studied. The obtained micro-PL spectra are shown in Fig. 5. Under low excitation well-resolved sharp emission lines are observed. With the increase in laser power intensity the $\mu$-PL spectrum changes into broad band. In the experiment no increase in the luminescence intensity of sharp line was observed, so we think that the increased generation of carriers, since they filled up the allowed states, does not influence the single dot any more. We suppose that 
rather further dots are excited and the luminescence from adjacent dots and/or deeper layers is observed. It can be predicted that the energy shift — blue because of states filling and electric field screening, or red - caused by the band gap renormalization effect, should be visible. In the applied excitation power no significant changes in the emission energy from the QDs was observed.

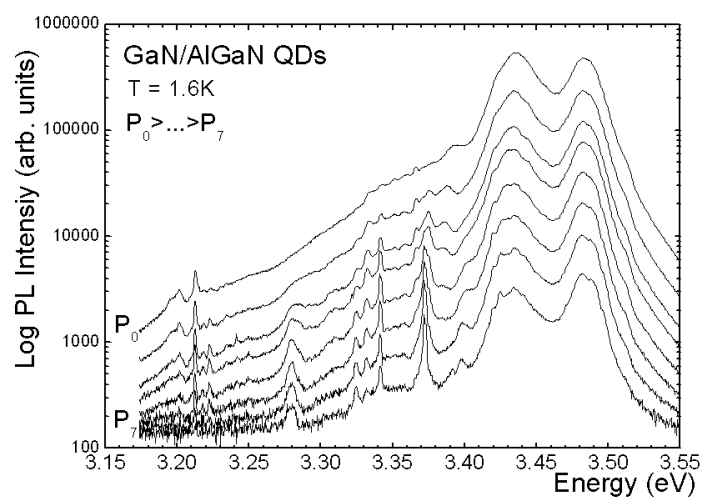

Fig. 5. Micro-photoluminescence spectra of GaN/AlGaN QDs measured with different excitation powers.

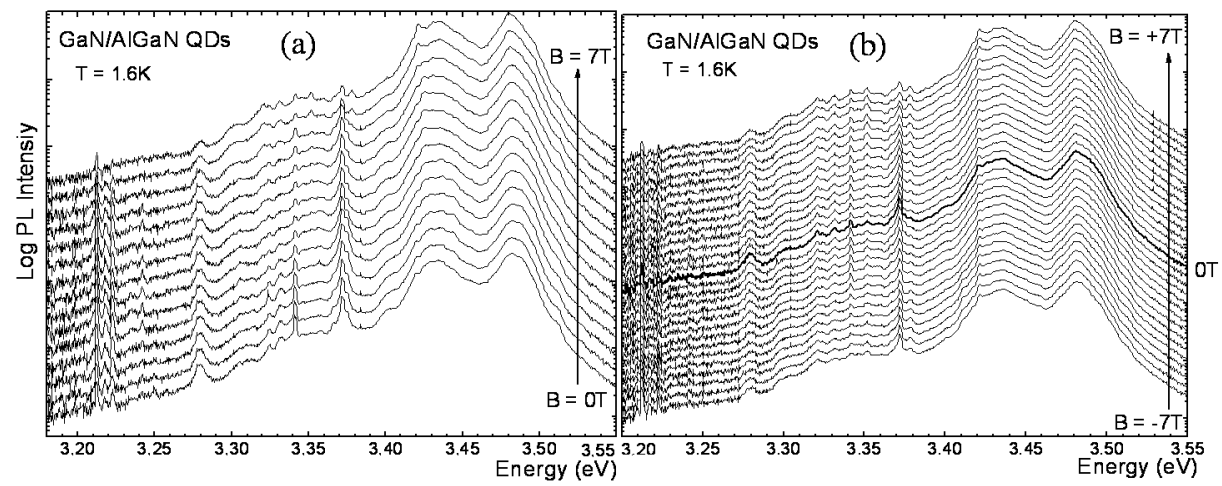

Fig. 6. Micro-magneto-photoluminescence of GaN/AIGaN QDs collected at different sample spots, (a) spectra up to $7 \mathrm{~T}$, (b) spectra up to $7 \mathrm{~T}$ in two circular polarizations.

In order to investigate the magnetic properties of the QDs and find the orbital symmetry of the energy levels we carried out the magnetic field dependent $\mu$-PL measurements. The magnetic field evolution of the spectra is show in Fig. $6 \mathrm{a}$ up to $7 \mathrm{~T}$ and Fig. $6 \mathrm{~b}$ up to $7 \mathrm{~T}$ for two circular polarizations. It appeared that up to $7 \mathrm{~T}$, the magnetic field does not have a great influence on the measured spectra. Even in the polarization resolved experiment no significant shifts of the emission energy is observed and the effects are polarization independent. It thus 
can be concluded that the accessible magnetic fields are not sufficient to influence the electronic states and to observe any changes in the experiment.

\section{Summary}

We have presented a new possibility to grow low density GaN/AlGaN quantum dots identified by AFM. For such structures we observed the sharp emission lines in the micro-photoluminescence experiments. To the best authors' knowledge such emission that can be attributed to the luminescence from individual quantum dots in nitride based structures is reported for the first time. The obtained results can serve as a background for further studies of electronic structure of individual QDs in GaN/AlGaN system.

\section{References}

[1] S. Tanaka, S. Iwai, Y. Aoyagi, Appl. Phys. Lett. 69, 4096 (1996).

[2] X.-Q. Shen, S. Tanaka, S. Iwai, Y. Aoyagi, Appl. Phys. Lett. 72, 344 (1996).

[3] V. Dimitriev, K. Irvine, A. Zubrilov, D. Tsvetkov, V. Nikolaev, M. Jakobson, D. Nelson, A. Sitnikova, in: Gallium Nitride and Related Materials, Eds. R.D. Dupuis, J.A. Edmond, F.A. Ponce, S. Nakamura, Materials Research Society, Pittsburgh (PA) 1996

[4] P. Ramvall, S. Tanaka, S. Nomura, P. Riblet, Y. Aoyagi, Appl. Phys. Lett. 73, 1104 (1998); R. Ramvall, P. Riblet, S. Nomura, Y. Aoyagi, J. Appl. Phys. 87, 3883 (2000).

[5] K. Pakuła, R. Bożek, J.M. Baranowski, J. Jasinski, Z. Liliental-Weber, J. Cryst. Growth 267, 1 (2004).

[6] S.-E. Park, S.-M. Lim, C.-R. Lee, C.S. Kim, B.O, J. Cryst. Growth 249, 487 (2003).

[7] J. Jasny, J. Sepiol, T. Irngartinger, M. Traber, A. Renn, U.P. Wild, Rev. Sci. Instrum. 67, 1425 (1996).

[8] J.A. Gaj, A. Golnik, P. Kosacki, K. Kowalik, W. Maslana, M. Kutnowski, T. Wojtowicz, Phys. Status Solidi C 1, 831 (2004).

[9] E. Martinez-Guerrero, C. Adelmann, F. Chaubel, J. Simon, N.T. Pelekanos, G. Mula, B. Daudin, G. Feuillet, H. Mariette, Appl. Phys. Lett. 77, 809 (2000). 